\title{
Comprehensive, in-hospital geriatric assessment plus an interdisciplinary home intervention after discharge reduced length of subsequent readmissions and improved functioning
}

\author{
Nikolaus T, Specht-Leible N, Bach M, et al. A randomized trial of comprehensive geriatric assessment and home \\ intervention in the care of hospitalized patients. Age Ageing 1999 Oct;28:543-50.
}

QUESTION: Is an in-hospital, comprehensive geriatric assessment alone or combined with an interdisciplinary home intervention after discharge more effective than usual care?

Source of funding: Sozialministerium Baden Wïrttemberg.

For correspondence: Dr T Nikolaus, Department of Geriatric Medicine, University of Ulm and Bethesda Geriatric Clinic, Zollernring 26-28, D-89073 Ulm, Germany. Fax +490731187387

\section{Setting}

A university affiliated geriatric hospital in Heidelberg, Germany and patient homes.

\section{Patients}

545 patients (mean age 81 y, 73\% women) who were admitted from home with acute illness and had multiple chronic conditions or functional deterioration after convalescence, or were at risk of nursing home placement. Patients with terminal illness or severe dementia were excluded. Follow up at 1 year was $94 \%$.

\section{Intervention}

181 patients were allocated to comprehensive, geriatric assessment plus in-hospital and post-discharge treatment at home by an interdisciplinary team, which consisted of 3 nurses, a physiotherapist, an occupational therapist, and a social worker (home intervention group). The in-hospital, comprehensive assessment addressed activities of daily living, cognition, social situation, and perceived health. The team provided additional treatment while the patient was in hospital (eg, additional training in dressing or walking). Before discharge, the team made 1 home visit to assess the patient's home and prescribe technical aids as necessary. After discharge, the team provided treatment that home services could not, or could not immediately, provide. 179 patients were allocated to comprehensive assessment alone, and 185 were allocated to usual care, which comprised an assessment of activities of daily living and cognition.

\section{Main outcome measures}

Main outcomes were mortality, hospital readmission, nursing home placement, functional status (Barthel index), and direct costs (staff, use of community services, hospital and physician visits, and nursing home days).

\section{Main results}

Analysis was by intention to treat. The 3 groups did not differ for mortality at 1 year (table). Among the survivors $(\mathrm{n}=420)$, the home intervention group had shorter stays during geriatric hospital readmissions $(\mathrm{p}<0.05)$, shorter nursing home placements $(\mathrm{p}<0.05)$, and better instrumental functioning $(\mathrm{p}<0.05)$ (table). The home intervention group did not differ from the assessment and usual care groups for number of hospital readmissions or admissions to nursing homes. The average annual net savings per person in the home care group was US $\$ 4000$.

\section{Conclusion}

Among elderly patients in hospital with acute illness, comprehensive geriatric assessment plus an interdisci-

plinary home visit after discharge reduced the duration of subsequent hospital readmissions and improved functional status, but did not affect mortality, readmission rate, or nursing home placements when compared with comprehensive assessment alone or usual care.

*Information provided by author.

Home intervention v assessment alone v usual care for elderly patients admitted to hospital with acute illness

\begin{tabular}{lccc} 
Outcomes at 1 year & $\begin{array}{l}\text { Home } \\
\text { intervention }\end{array}$ & $\begin{array}{l}\text { Assessment } \\
\text { alone }\end{array}$ & $\begin{array}{c}\text { Usual } \\
\text { care }\end{array}$ \\
\hline Mortality $\dagger$ & $18.2 \%$ & $16.8 \%$ & $17.3 \%$ \\
\hline $\begin{array}{l}\text { Length of stay during geriatric hospital } \\
\text { readmission, days }\end{array}$ & 15.3 & 32.3 & 34.0 \\
\hline Length of nursing home stay, days & 114.7 & 161.6 & 170.0 \\
\hline Instrumental functioning score & 5.6 & 4.1 & 4.3 \\
\hline
\end{tabular}

$\dagger$ No significant difference among groups.

\section{COMMENTARY}

With a growing population of people $>65$ years of age, there is an increasing focus on identifying and evaluating interventions that maintain elderly people in their own homes for as long as possible. This study examined whether an in-hospital, comprehensive geriatric assessment alone or combined with an interdisciplinary home intervention after discharge would affect mortality, functional status, rehospitalisation, nursing home placement, and direct costs.

The results of this study support the work done in the Program for All-inclusive Care of the Elderly (PACE), begun in San Francisco. ${ }^{1}$ By using a comprehensive assessment that addresses both functional and medical concerns, care can be coordinated and provided to meet the needs of older people. However, of note in the study by Nikolaus et al is the finding that comprehensive geriatric assessment alone did not improve outcomes. The addition of a home intervention team accounted for improvement in outcomes. These findings can help gerontological healthcare providers to rethink approaches to providing care to frail, elderly people.

The authors reported that intervention patients' increased knowledge about and use of community services resulted in increased client satisfaction and health status perception. Improved communication among healthcare team members contributed to the reduced length of hospital stay. The additional costs for the home intervention team and increased use of community services by their patients were more than offset by the savings resulting from the reduced number of days spent in hospital and nursing homes.

Because the same physicians provided care to patients in all groups, possible improved care of control patients could have contaminated the data (a study limitation noted by the authors). It is also possible that the 1 year follow up may have been too short to show a difference in mortality.

Nurses in home care, acute care, or long term care settings can use the findings of this study to improve their practice. Providing comprehensive care requires a collaborative effort that includes effective communication skills. Frail, elderly clients can benefit from this care by realising that their individual needs are considered and met. This will lead to increased client satisfaction with life and health.

Suzanne K Beltz, RN, PhD, CS, CNS Associate Professor, Clinical Specialist in Gerontological Nursing University of Mary Hardin-Baylor

1 Kane RL. Contemporary Gerontology 1999;6:47-50. 